\title{
The role of surfactants on the mechanism of the long-wave instability in liquid film flows
}

\author{
George Karapetsas $\dagger$ and Vasilis Bontozoglou \\ Department of Mechanical Engineering, University of Thessaly, GR-38334 Volos, Greece
}

(Received 28 June 2013; revised 30 September 2013; accepted 16 December 2013)

The analysis for the physical mechanism of the long-wave instability in liquid film flow is extended to take into account the presence of a surfactant of arbitrary solubility. The Navier-Stokes equations are supplemented by mass balances for the concentrations at the interface and in the bulk, by a Langmuir model for adsorption kinetics at the interface, and are expanded in the limit of long-wave disturbances. The longitudinal flow perturbation, known to result from the perturbation shear stress which develops along the deformed interface, is shown to contribute a convective flux that triggers an interfacial concentration gradient. This gradient is, at leading order, in phase with the interfacial deformation, and as a result produces Marangoni stresses that stabilize the flow. The strength of the interfacial concentration gradient is shown to be maximum for an insoluble surfactant and to decrease with increasing surfactant solubility. The decrease is explained in terms of the spatial phase of mass transfer between interface and bulk, which mitigates the interfacial flux by the flow perturbation and leads to the attenuation of Marangoni stresses. Higher-order terms are derived, which provide corrections for disturbances of finite wavelength.

Key words: instability, interfacial flows (free surface), Marangoni convection

\section{Introduction}

The formation of waves in gravity-driven liquid films flowing down inclined surfaces has attracted the interest of many researchers in the past because of its importance in a broad range of engineering applications. Extensive reviews of the rich dynamics of this system and of the recent developments in the field are offered by Chang (1994), Oron, Davis \& Bankoff (1997), Craster \& Matar (2009) and Kalliadasis et al. (2012). It is well known that interfacial instabilities can be significantly affected by the presence of surface-active materials or surfactants (see for example Hameed et al. 2008; Conroy et al. 2011). Wave formation in falling films is no exception, and early experimental studies (see e.g. Emmert \& Pigford 1954; Stirba \& Hurt 1955; Tailby \& Portalski 1961) showed that the addition of even small amounts of surfactants can have a stabilizing influence on the flow, dampening the waves that would otherwise arise.

Predictions of the critical Reynolds number for the onset of waves on a clean liquid film (without surfactant) were first provided by Benjamin (1957) and Yih (1963), but a

$†$ Email address for correspondence: gkarapetsas@gmail.com 
full understanding of the physical mechanism responsible for this long-wave instability was achieved much later (Kelly et al. 1989; Smith 1990), by extending an argument originally proposed by Hinch (1984). It is notable that an unequivocal experimental confirmation of the linear prediction was also delayed significantly (Liu, Paul \& Gollub 1993), and that an unexpected strong effect of the channel width was reported very recently by Georgantaki et al. (2011) and Pollak, Haas \& Aksel (2011).

The primary instability of liquid films doped with insoluble surfactants (i.e. surfactants that do not dissolve to any significant effect in the bulk, but are assumed to reside only on the interface) was first considered by Benjamin (1964) and Whitaker (1964). They both predicted that insoluble surfactants delay the instability because of the elasticity they contribute to the interface. More specifically, surface tension depends on the concentration of surfactant, thus interfacial concentration gradients produce stresses (Marangoni stresses) that are responsible for the elastic behaviour. The role of surface elasticity was confirmed repeatedly in the literature (Anshus \& Acrivos 1967; Lin 1970; Blyth \& Pozrikidis 2004; Pereira \& Kalliadasis $2008 a$ ), and the mechanism responsible for the modified instability was investigated by Wei $(2005 a, b)$ for the case of insoluble surfactants.

Soluble surfactants exhibit more complex behaviour, because interfacial dynamics is intricately coupled with mass exchange with the bulk. Mass transfer rates, as well as the kinetics of adsorption, have been shown to be critical in the special case of a volatile soluble surfactant, which actually enhances instability (Ji \& Setterwall 1994; Shkadov, Velarde \& Shkadova 2004). Enhancement of instability was also shown by Yiantsios \& Higgins (2010) to be possible under conditions of evaporating thin films in the presence of non-volatile soluble surfactants. The general problem of the linear stability of non-evaporating film flow doped with a non-volatile soluble surfactant was considered recently (Karapetsas \& Bontozoglou 2013), and the critical Reynolds number was predicted as a function of surfactant solubility. However, a full mechanistic understanding of the role of a soluble surfactant appears to be missing.

Thus, the scope of the present study is to describe in detail the role of a surfactant of arbitrary solubility in the initiating and the growth mechanism of the long-wave instability in liquid film flow. The study constitutes a direct extension of the work of Smith (1990), and also confirms and complements the findings of Karapetsas \& Bontozoglou (2013).

\section{Problem formulation and scaling}

We study the dynamics of liquid films flowing along an infinite planar wall, inclined at an angle $\alpha$ with the horizontal plane (see figure 1). The film is laden with a soluble, non-volatile surfactant which may adsorb at the liquid-air interface altering surface tension, or it can exist in the bulk in the form of monomers. The fluid is Newtonian with constant density $\rho$ and kinematic viscosity $v$, and with surface tension, $\sigma$, which depends on the interfacial concentration of surfactant, $\Gamma$, through a constitutive equation $\sigma=\sigma(\Gamma)$. For the present purposes of a linear analysis, we do not have to consider a specific constitutive law for surface tension. We define, however, the surface elasticity $E(\Gamma)$

$$
E(\Gamma)=-\frac{\mathrm{d} \sigma}{\mathrm{d}(\ln \Gamma)}
$$

which contains the dependence of surface tension on interfacial surfactant concentration; a specific expression for $E(\Gamma)$ can be derived once the constitutive equation is specified. 


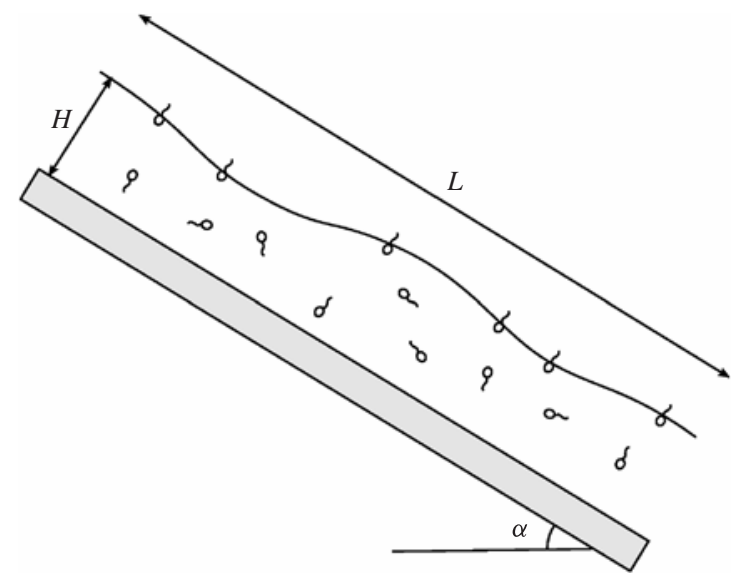

FIGURE 1. Schematic of a falling film in the presence of soluble surfactants.

In order to model two-dimensional dynamics, we use a Cartesian coordinate system $(x, z)$, with $x$ pointing in the streamwise and $z$ in the cross-stream direction. The velocity and pressure fields are $\boldsymbol{u}=(u, w)$ and $p$, respectively. The liquid-air interface is located at $z=h(x, t)$ and the liquid-solid interface at $z=0$. The flow is governed by the continuity and momentum conservation equations, subject to the zero-velocity boundary condition at the solid wall and the kinematic and dynamic boundary conditions at the free surface. The latter includes both a normal contribution due to surface tension and a tangential one due to the variation of surface tension along the free surface.

To account for the presence of soluble surfactants, we utilize a simplified version of the kinetic model of Edmonstone, Craster \& Matar (2006) and Karapetsas, Craster \& Matar $(2011 a, b)$. Surfactant partitioning between the bulk concentration, $c$, and the interfacial one, $\Gamma$, is described by a Langmuir model, with $\Gamma_{\infty}$ the interfacial concentration at maximum packing and $k_{1}, k_{2}$ the constants of the first-order adsorption and desorption kinetics. Mass conservation of adsorbed and dissolved surfactant is modelled by two advection-diffusion equations, one for the interface and one for the bulk. The net flux of monomer between interface and bulk is given by the kinetic model, and appears as a term in the equation of the interface and as a boundary condition in the equation of the bulk. The above physical model is the same as in Karapetsas \& Bontozoglou (2013), to which we refer the reader for a more detailed description.

The governing equations and boundary conditions are made dimensionless, using the following scaling:

$$
\begin{gathered}
(\tilde{x}, \tilde{z})=(x, z) / H, \quad \tilde{h}=h / H, \quad \tilde{t}=t U / H, \quad \tilde{\boldsymbol{u}}=\boldsymbol{u} / U \\
p=p_{a i r}+\rho g H \sin \alpha \tilde{p}, \quad(\tilde{\Gamma}, \tilde{c})=(\Gamma, H c) / \Gamma_{\infty}, \\
\tilde{J}_{b a}=J_{b a} \frac{H}{U \Gamma_{\infty}}, \quad \tilde{\sigma}=\frac{\sigma}{\sigma_{c}}, \quad \tilde{M}_{t o t}=\frac{M_{t o t}}{L \Gamma_{\infty}}
\end{gathered}
$$

Here $J_{b a}$ denotes the net flux of surfactant at the interface and $M_{t o t}$ is the total amount of surfactant that is present in our system. As a characteristic length we use the height of the Nusselt flat film, $H=(3 v Q /(g \sin \alpha))^{1 / 3}$, where $Q$ denotes 
the imposed flow rate. The velocities are scaled with the corresponding interfacial velocity, $U=g H^{2} \sin \alpha /(2 v)$, and $\sigma_{c}=\sigma(\Gamma=0)$ is the surface tension of the clean liquid. Tildes denote dimensionless variables and are henceforth suppressed.

Introducing the above scaling, the continuity and momentum conservation equations become,

$$
\begin{gathered}
u_{x}+w_{z}=0 . \\
\operatorname{Re}\left(u_{t}+u u_{x}+w u_{z}\right)+2 p_{x}-u_{x x}-u_{z z}-2=0, \\
\operatorname{Re}\left(w_{t}+u w_{x}+w w_{z}\right)+2 p_{z}-w_{x x}-w_{z z}+2 \cot \alpha=0,
\end{gathered}
$$

where $R e=g H^{3} \sin \alpha / 2 v^{2}$ is the Reynolds number. Unless stated otherwise, the subscripts denote partial differentiation with respect to $x, z$ and time $t$.

The components of the dynamic boundary condition tangential and normal to the free surface are, respectively,

$$
\begin{aligned}
-4 u_{x} h_{x}+\left(u_{z}+w_{x}\right)\left(1-h_{x}^{2}\right) & =2 W e \sigma_{x} \sqrt{1+h_{x}^{2}}, \\
p+\frac{u_{x}\left(1-h_{x}^{2}\right)+\left(u_{z}+w_{x}\right) h_{x}}{1+h_{x}^{2}} & =-W e \sigma \frac{h_{x x}}{\left(1+h_{x}^{2}\right)^{3 / 2}}
\end{aligned}
$$

where $W e=\sigma_{c} / \rho g H^{2} \sin \alpha$ is the Weber number. In addition, we impose at the liquidsolid interface $(z=0)$, the usual no-slip, no-penetration conditions, $u=w=0$, and along the moving interface $(z=h(x, t))$ the kinematic boundary condition $h_{t}+u h_{x}=w$.

The dimensionless form of surfactant conservation in the bulk becomes

$$
c_{t}+u c_{x}+w c_{z}=P e_{b}^{-1}\left(c_{x x}+c_{z z}\right),
$$

where $P e_{b}=\operatorname{Rev} / D_{b}$ is the Péclet number in the bulk, with $D_{b}$ the surfactant diffusivity in the bulk. The above equation is subject to the following boundary conditions, along the interface $(z=h(x, t))$

$$
\frac{h_{x} c_{x}-c_{z}}{P e_{b} \sqrt{1+h_{x}^{2}}}=J_{b a}
$$

and along the wall $(z=0)$

$$
c_{z}=0
$$

Surfactant conservation at the interface is modelled by the following advectiondiffusion equation

$$
\Gamma_{t}+u \Gamma_{x}+\Gamma \nabla_{s} \cdot \boldsymbol{u}=\frac{\nabla_{s}^{2} \Gamma}{P e_{a}}+J_{b a},
$$

where $P e_{a}=\operatorname{Rev} / D_{a}$ is the interfacial Péclet number, with $D_{a}$ the surfactant diffusivity at the interface, and $\nabla_{s}$ is the surface gradient, defined as $\nabla_{s}=\left(I-\boldsymbol{n n}^{T}\right) \cdot \nabla$. It is noted that the derivation of (2.11) involves some subtle points related to the movement of the interface and the definition of the time derivative (Pereira \& Kalliadasis $2008 b$ ). Another version of this equation has been repeatedly quoted incorrectly in the literature but as was shown by Pereira \& Kalliadasis (2008b), equation (2.11) is actually more appropriate. is

Finally, the dimensionless expression for the net adsorption flux in (2.9) and (2.11)

$$
J_{b a}=k_{a}\left[\left.\xi_{a} c\right|_{z=h}(1-\Gamma)-\Gamma\right] .
$$


where $k_{a}=k_{2} H / U$ and $\xi_{a}=k_{1} / k_{2} H$. The physical significance of these parameters is as follows: Term $k_{a}$ is the ratio of the time scale of convection to the time scale of desorption. For example, $k_{a} \ll 1$ means that the backward reaction is slow compared with convection, and thus a temporary decrease of the bulk concentration near the interface will not lead to significant desorption. Term $\xi_{a}$ is the ratio of the kinetic constants of the forward and backward reaction for the interface-bulk interaction, and thus provides a direct measure of surfactant solubility in the bulk (Jensen \& Grotberg 1993). More specifically, $\xi_{a} \ll 1$ signifies a highly soluble and $\xi_{a} \gg 1$ a sparingly soluble surfactant.

\section{Linearization and normal mode analysis}

The above set of governing equations and boundary conditions has a trivial solution corresponding to a flat film with uniform surfactant concentration. In the case of a clean fluid, this is also known as the Nusselt flat film solution and its dimensionless form is shown below

$$
\bar{h}=1, \quad \bar{u}=2 z-z^{2}, \quad \bar{w}=0, \quad \bar{p}=(1-z) \cot \alpha,
$$

where an overbar denotes the base state. In order for the above solution to remain valid when a soluble surfactant is present, the concentrations should be uniform and at equilibrium. Imposing these conditions leads to

$$
\bar{c}=\frac{\bar{\Gamma}}{\xi_{a}(1-\bar{\Gamma})},
$$

and given that the total mass of the surfactant, $M_{t o t}$, is conserved and evaluated by the following expression

$$
\bar{c}+\bar{\Gamma}=M_{t o t},
$$

it is possible to derive an analytical expression for $\bar{c}$ and $\bar{\Gamma}$ as function of the parameters $M_{\text {tot }}$ and $\xi_{a}$ (Karapetsas \& Bontozoglou 2013).

We perform a linear stability analysis by perturbing the flow around the above flat film solution. To this end we write all variables as the sum of the base state and a small perturbation

$$
\phi=\bar{\phi}+\phi^{\prime},
$$

and linearize the system assuming $\phi^{\prime} \ll \bar{\phi}$. We also apply the relevant boundary conditions around the mean elevation of the interface, $z=1$, by expanding the variables in Taylor series as follows

$$
\left.\phi\right|_{z=h}=\left.\bar{\phi}\right|_{z=h}+\left.\phi^{\prime}\right|_{z=h}=\left(\left.\bar{\phi}\right|_{z=1}+\left.h^{\prime} \frac{\partial \bar{\phi}}{\partial z}\right|_{z=1}\right)+\left.\phi^{\prime}\right|_{z=1}+O\left(h^{\prime 2}\right) .
$$

The set of linearized equations and boundary conditions is analysed by considering the normal modes:

$$
\left(\begin{array}{c}
h^{\prime}(x, t) \\
u^{\prime}(x, z, t) \\
w^{\prime}(x, z, t) \\
p^{\prime}(x, z, t) \\
c^{\prime}(x, z, t) \\
\Gamma^{\prime}(x, t) \\
J_{b a}^{\prime}(x, t)
\end{array}\right)=\left(\begin{array}{c}
\hat{h} \\
\hat{u}(z) \\
\hat{w}(z) \\
\hat{p}(z) \\
\hat{c}(z) \\
\hat{\Gamma} \\
\hat{J}_{b a}
\end{array}\right) \exp [\mathrm{i} k(x-C t)]
$$


where $k=2 \pi H / \lambda$ is the dimensionless wavenumber of the disturbance with wavelength $\lambda$, and $C$ is the complex eigenvalue, $C=C_{r}+\mathrm{i} C_{i}$, with $C_{r}$ the dimensionless phase velocity scaled with the Nusselt film surface velocity $U$ and $k C_{i}$ the growth rate. The final system of normal-mode disturbance equations is

$$
\begin{gathered}
\operatorname{Re}\left[\mathrm{i} k(\bar{u}-C) \hat{u}+\bar{u}_{z} \hat{w}\right]+2 \mathrm{i} k \hat{p}+k^{2} \hat{u}-\hat{u}_{z z}=0, \\
\mathrm{i} k \operatorname{Re}(\bar{u}-C) \hat{w}+2 \hat{p}_{z}+k^{2} \hat{w}-\hat{w}_{z z}=0, \\
\mathrm{i} k \hat{u}+\hat{w}_{z}=0, \\
P e_{b} \mathrm{i} k(\bar{u}-C) \hat{c}+k^{2} \hat{c}-\hat{c}_{z z}=0,
\end{gathered}
$$

and the following boundary conditions are applied along the mean interfacial elevation $(z=1)$

$$
\begin{gathered}
\hat{u}_{z}(1)+\mathrm{i} k \hat{w}(1)+\bar{u}_{z z}(1) \hat{h}+2 \mathrm{i} k W e \frac{\bar{E}}{\bar{\Gamma}} \hat{\Gamma}=0, \\
\hat{p}(1)-\hat{h} \cot \alpha+\mathrm{i} k \hat{u}(1)-k^{2} W e \bar{\sigma} \hat{h}=0, \\
\mathrm{i} k[\bar{u}(1)-C] \hat{h}-\hat{w}(1)=0, \\
(\bar{u}(1)-C) \mathrm{i} k \hat{\Gamma}+\bar{\Gamma} \mathrm{i} k \hat{u}(1)+\frac{k^{2} \hat{\Gamma}}{P e_{a}}-\hat{J}_{b a}=0, \\
\hat{J}_{b a}=\frac{-\hat{c}_{z}(1)}{P e_{b}}=k_{a}\left[\xi_{a}[(1-\bar{\Gamma}) \hat{c}(1)-\bar{c} \hat{\Gamma}]-\hat{\Gamma}\right],
\end{gathered}
$$

and along the wall $(z=0)$

$$
\begin{gathered}
\hat{u}(0)=\hat{w}(0)=0, \\
\hat{c}_{z}(0)=0 .
\end{gathered}
$$

In the above $\bar{\sigma}$ denotes the surface tension that corresponds to the base state surface concentration $\bar{\Gamma}$ and

$$
\bar{E}=-\bar{\Gamma}\left(\frac{\mathrm{d} \bar{\sigma}}{\mathrm{d} \bar{\Gamma}}\right)_{\bar{\Gamma}} .
$$

\section{Long-wave expansion}

\subsection{Initiating mechanism}

The physical system considered has been shown (Karapetsas \& Bontozoglou 2013) to be susceptible to a long-wave instability. The mechanism for this instability can be identified in the ordered problems that arise from a regular expansion of the normalmode amplitudes in the limit $k \rightarrow 0$. More specifically, we posit

$$
\begin{gathered}
\hat{u}=u_{0}+k u_{1}+k^{2} u_{2}+O\left(k^{3}\right), \\
\hat{w}=k w_{1}+k^{2} w_{2}+O\left(k^{3}\right), \\
\hat{p}=p_{0}+k p_{1}+k^{2} p_{2}+O\left(k^{3}\right), \\
C=C_{0}+k C_{1}+k^{2} C_{2}+O\left(k^{3}\right), \\
\hat{c}=c_{0}+k c_{1}+k^{2} c_{2}+O\left(k^{3}\right), \\
\hat{\Gamma}=\Gamma_{0}+k \Gamma_{1}+k^{2} \Gamma_{2}+O\left(k^{3}\right), \\
\hat{J}_{b a}=J_{b a, 0}+k J_{b a, 1}+k^{2} J_{b a, 2}+O\left(k^{3}\right), \\
\hat{h}=h_{0}+k h_{1}+k^{2} h_{2}+O\left(k^{3}\right) .
\end{gathered}
$$


We are interested in the 'interfacial mode' that is triggered by a deflection of the free surface, and which is known to be the unstable one (Blyth \& Pozrikidis 2004; Pereira \& Kalliadasis 2008a); the aforementioned papers have shown for an insoluble surfactant that a 'concentration mode' also exists, which is stable and actually not related to the surfactant property (see Appendix). Thus, we normalize the system of equations by assuming that $h_{0}=1$ and $h_{1}=h_{2}=0$, implying that all perturbation amplitudes to be subsequently calculated are proportional to the amplitude, $h_{0}$, of the free surface deformation. The lack of an $O\left(k^{0}\right)$ term in the expansion of $\hat{w}$ in the long-wave limit is a direct consequence of continuity, (3.9).

At order $O\left(k^{0}\right)$, we combine the normal mode momentum equations, the force balance at the interface and the wall boundary condition,

$$
u_{0 z z}=0, \quad u_{0}(0)=0, \quad u_{0 z}(1)=-\bar{u}_{z z}(1)=2, \quad p_{0 z}=0, \quad p_{0}(1)=\cot \alpha,
$$

and obtain the well-known leading-order perturbation to the base flow,

$$
u_{0}(z)=2 z, \quad p_{0}(z)=\cot \alpha .
$$

As explained by Kelly et al. (1989) and Smith (1990), when the interface deforms, a non-zero shear stress, $\bar{u}_{z z}(1) h^{\prime}$, results from the base flow because of the local curvature of its velocity profile at the interface. Therefore, a perturbation shear stress develops that exactly cancels the above, and this drives the longitudinal flow perturbation $u_{0}(z)$.

In order to solve for the concentrations, we combine at order $O\left(k^{0}\right)$ mass conservation in the bulk and at the interface, and no-penetration at the wall, and obtain

$$
c_{0 z z}=0, \quad c_{0 z}(0)=0, \quad \frac{c_{0 z}(1)}{P e_{b}}=-J_{b a, 0} .
$$

Therefore,

$$
c_{0}(z)=\frac{\Gamma_{0}}{\xi_{a}(1-\bar{\Gamma})^{2}}=\text { constant. }
$$

Equations (4.11) and (4.12) state that, at zero order, convection is negligible and the interface is in equilibrium with the bulk. This is reasonable, given that in the limit $k \rightarrow 0$ variations along the wavelength become very slow, and the resistance to mass transfer in the cross-stream direction diminishes.

It is also notable that the concentrations are not fully determined at order $O\left(k^{0}\right)$. This happens because the initial deformation of the interface does not trigger by itself any change in the concentration of surfactant (the dilation of the interface is second order in the deformation amplitude, i.e. linearly negligible). As will be shown next, it is actually the leading-order flow perturbation, $u_{0}(1)$, that disturbs the interfacial concentration, and this occurs through a convective contribution that appears at order $O\left(k^{1}\right)$.

Next, we consider the normal mode, mass conservation equations at order $O\left(k^{1}\right)$ and obtain

$$
P e_{b} i\left(\bar{u}-C_{0}\right) c_{0}=c_{1 z z}, \quad c_{1 z}(0)=0, \quad \frac{c_{1 z}(1)}{P e_{b}}=-J_{b a, 1},
$$

and

$$
\left(\bar{u}(1)-C_{0}\right) \mathrm{i} \Gamma_{0}+\mathrm{i} \bar{\Gamma} u_{0}(1)=-\frac{c_{1 z}(1)}{P e_{b}} .
$$


The leading-order wave velocity, $C_{0}$, is derived from the kinematic boundary condition (3.13),

$$
\mathrm{i}\left(\bar{u}(1)-C_{0}\right)=w_{1}(1) \Rightarrow C_{0}=\bar{u}(1)+\mathrm{i} w_{1}(1) .
$$

From continuity (3.9), we obtain

$$
w_{1 z}+\mathrm{i} u_{0}=0 \Rightarrow w_{1}(z)=-\mathrm{i} z^{2},
$$

and therefore we deduce that

$$
C_{0}=2 .
$$

Integrating (4.13), we obtain the $O\left(k^{1}\right)$ amplitude of the bulk concentration perturbation

$$
c_{1}(z)=\mathrm{i} c_{0}\left[P e_{b}\left(\frac{3}{4}-z^{2}+\frac{z^{3}}{3}-\frac{z^{4}}{12}\right)+\frac{4}{3(1-\bar{\Gamma}) k_{a} \xi_{a}}-\mathrm{i} \frac{\Gamma_{1}}{\Gamma_{0}}\right] .
$$

Equation (4.14) may now be solved, using (4.12), (4.17) and (4.18), to obtain the zero-order perturbation in the interfacial concentration of surfactant,

$$
\Gamma_{0}=2 \bar{\Gamma}\left(\frac{3 \xi_{a}(1-\bar{\Gamma})^{2}}{3 \xi_{a}(1-\bar{\Gamma})^{2}+4}\right),
$$

and, through (4.12), also the zero-order perturbation to the bulk concentration.

Analysis of (4.14) provides an understanding of the initiating mechanism of concentration perturbation. We focus first on the two terms on the left-hand side, which balance exactly in the case of an insoluble surfactant. The second term represents convective transport of the mean interfacial concentration because of the variation along the wave of the leading-order flow perturbation, $u_{0}(1)$. This term disrupts the uniform interfacial concentration; more specifically, it produces a flux that is maximum at the back node and minimum at the front node ('back' and 'front' are in reference to the crest of surface displacement), and as a result, it moves surfactant away from the trough and towards the crest. Thus, a perturbation $\Gamma_{0}$ with maximum at the crest and minimum at the trough arises, whose convective transport by the mean flow is expressed by the first term on the left-hand side of (4.14). The perturbation in $\Gamma$ is such that the two convective fluxes, combined with mass exchange with the bulk (the right-hand side term), balance. We should note at this point that in the case of insoluble surfactants, the concentration was shown by Wei (2005b) to be in phase with the surface displacement and we see that, at leading order, this is true for soluble surfactants as well.

Equivalently, we may consider a reference frame that renders the wave stationary, and in this case the interfacial velocity is given by

$$
\left.u\right|_{z=h}-C_{0}=\left[\bar{u}(1)-C_{0}\right]+u^{\prime}(1),
$$

and since $u^{\prime}(1)=\hat{u}(1) h^{\prime}=u_{0}(1) h^{\prime}+O\left(k^{2}\right)$ and $u_{0}(1)=2$ we obtain

$$
\left.u\right|_{z=h}-C_{0}=-1+2 h^{\prime}+O\left(k^{2}\right) .
$$

Therefore, the interfacial streamwise velocity has a magnitude which is maximum at the trough and minimum at the crest of the interfacial displacement (see figure 2). Given that the system is at steady state in this reference frame (and thus the flux of surfactant is the same at every streamwise location), we conclude that the interfacial 


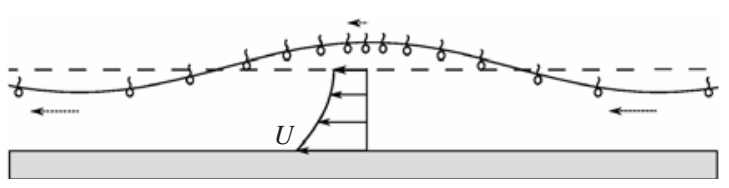

FIGURE 2. A disturbance to the free surface. The dotted arrows depict the direction and magnitude of the interfacial velocity at various positions, assuming a moving frame of reference that renders the wave stationary. The long-dashed line is the undisturbed free surface position and the line arrows depict the mean velocity profile in this reference frame.

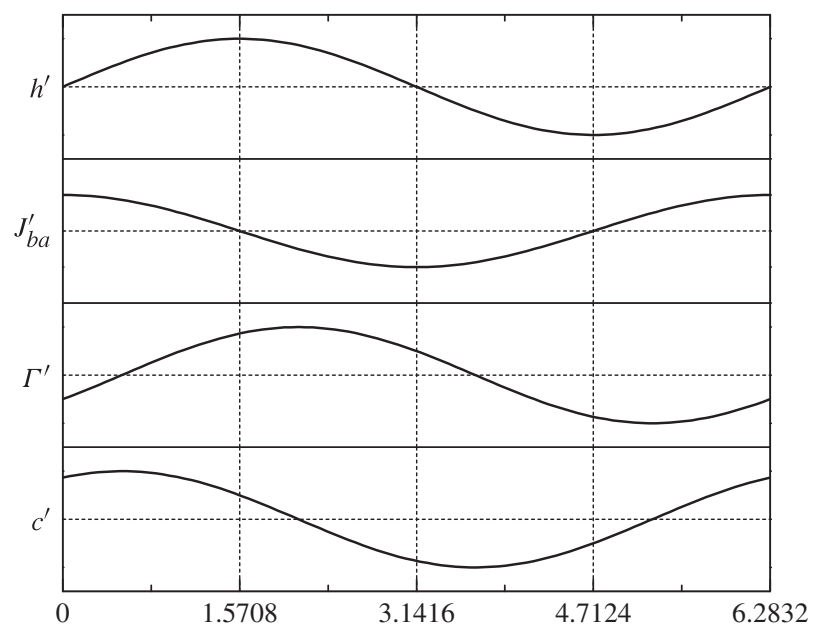

FIGURE 3. Spatial wave forms of the film height, $h^{\prime}$, interfacial flux, $J_{b a}^{\prime}$, interfacial concentration, $\Gamma^{\prime}$, and concentration in the bulk, $c^{\prime}$.

concentration varies inversely to the interfacial velocity, i.e. it is maximum at the crest and minimum at the trough.

It is noted that the three fluxes in (4.14) have the same phase, which lags $90^{\circ}$ behind the displacement (as will be shown below, $c_{1}(z)$ is pure imaginary). Therefore, the perturbation in surfactant concentration is at leading order in phase with the interface displacement, a result that is directly evident from (4.19); as will be shown later, the phases start to deviate at order $O\left(k^{1}\right)$. Equation (4.19), also indicates that the leading-order effect of surfactant solubility is to decrease the magnitude of concentration perturbation at the interface (it is recalled that $\xi_{a} \gg 1$ corresponds to a sparingly soluble, and $\xi_{a} \ll 1$ to a highly soluble surfactant).

The effect of surfactant solubility becomes more evident by evaluating directly the rate of mass transfer between the bulk and the interface, i.e. the right-hand side of (4.14). Using (4.18), we obtain

$$
J_{b a, 1}=-\frac{c_{1 z}(1)}{P e_{b}}=\frac{\mathrm{i} 8 \bar{\Gamma}}{3 \xi_{a}(1-\bar{\Gamma})^{2}+4} .
$$

We observe that the transfer rate of surfactant is $90^{\circ}$ out of phase with the displacement (see figure 3), i.e. at the front node (with respect to the crest) transfer of surfactant is from the interface towards the bulk, and at the back node it is from the 
bulk towards the interface. This is precisely the opposite effect from the convective transport by the leading-order flow perturbation, as described above. Thus, exchange with the bulk opposes transport by the flow perturbation, and therefore diminishes the perturbation in interfacial concentration of surfactant. In the limit of a highly soluble surfactant $\left(\xi_{a} \rightarrow 0\right) J_{b a, 1}=\mathrm{i} 2 \bar{\Gamma}$ and $\Gamma_{0}=0$, i.e. the two fluxes become exactly equal and opposite, and as a result interfacial gradients (and Marangoni stresses) disappear altogether.

\subsection{Effect of surfactant on the growth mechanism}

We proceed to calculate the $O\left(k^{1}\right)$ longitudinal velocity perturbation, $u_{1}(z)$, which is evaluated from the $x$-momentum equation (3.7)

$$
u_{1 z z}=2 \mathrm{i} p_{0}+\operatorname{Re}\left[\mathrm{i}\left(\bar{u}-C_{0}\right) u_{0}+\bar{u}_{z} w_{1}\right],
$$

subject to the boundary conditions of zero velocity at the wall, $u_{1}(0)=0$, and tangential force balance at the interface,

$$
u_{1 z}(1)=-2 \mathrm{i} \frac{W e \bar{E}}{\bar{\Gamma}} \Gamma_{0} .
$$

Equations (4.23) and (4.24) contain the essence of the growth mechanism. Following Smith (1990), we recall that (4.23) represents a balance between viscous stress gradient, $u_{1 z z}$, and the pressure and inertial stresses that contribute to the flow $u_{1}$. Term $p_{0}=\cot \alpha$ is positive, thus pressure is in phase with interface deformation, i.e. maximum below the crest and minimum below the trough. This distribution is stabilizing, draining liquid away from the crests and towards the troughs. In contrast, the two inertial stresses, representing advection of flow perturbations by the base state velocity relative to the moving disturbance, are negative and therefore destabilizing. In particular, the dominant negative contribution, $-\operatorname{Re} C_{0} u_{0}$, corresponds to temporal acceleration by the wave motion, whereas the remaining terms represent convective acceleration and sum up to a slightly stabilizing contribution. Thus, the unsteadiness associated with the moving disturbance is the cause of the instability.

The role of surfactant is given by the boundary condition (4.24), which represents the contribution to the flow $u_{1}$ due to Marangoni stresses. More specifically, integration of (4.23) subject to (4.24) gives

$$
u_{1}(z)=\mathrm{i}\left[\operatorname{Re}\left(\frac{z^{4}}{6}-\frac{2 z^{3}}{3}+\frac{4 z}{3}\right)-\cot \alpha\left(2 z-z^{2}\right)-4 W e \bar{E}\left(\frac{3 \xi_{a}(1-\bar{\Gamma})^{2}}{3 \xi_{a}(1-\bar{\Gamma})^{2}+4}\right) z\right],
$$

which indicates that the $O\left(k^{1}\right)$ longitudinal velocity perturbation is $90^{\circ}$ out of phase with the displacement, attaining extrema at the nodes of the travelling wave. The last term on the right-hand side of (4.25) represents the additional flow perturbation caused by the Marangoni stresses at the interface. Its coefficient is negative, and thus it is maximum at the front node and minimum at the back node with respect to the crest, i.e. it drives liquid away from the crest. This is the expected effect of the Marangoni stresses that are induced by the leading-order perturbation in $\Gamma$ (equation (4.19)) given that $\Gamma_{0}$ is in-phase with surface displacement, i.e. maximum at the crests and minimum at the troughs. The action of each of the terms of (4.25) is depicted schematically in figure 4 . 


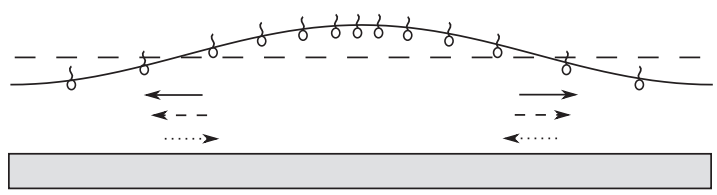

FIgURE 4. A disturbance to the free surface. The line arrow depicts the action of Marangoni stresses (stabilizing), the dashed arrow depicts the action of pressure (stabilizing) and the dotted arrow depicts the action of inertia terms (destabilizing) in (4.25). The long-dashed line is the undisturbed free-surface position.

The contribution to the complex eigenvalue at order $O\left(k^{1}\right)$ is determined from the kinematic boundary condition (3.13) at order $O\left(k^{2}\right)$

$$
C_{1}=\mathrm{i} w_{2}(1)
$$

The cross-stream velocity $w_{2}$ is calculated by integration of the continuity equation, $w_{2 z}+\mathrm{i} u_{1}=0$, subject to $w_{2}(0)=0$. Substituting $u_{1}$ from (4.25), we obtain

$$
w_{2}(z)=\cot \alpha\left(\frac{z^{3}}{3}-z^{2}\right)+\operatorname{Re}\left(\frac{2 z^{2}}{3}-\frac{z^{4}}{6}+\frac{z^{5}}{30}\right)-2 W e \bar{E}\left(\frac{3 \xi_{a}(1-\bar{\Gamma})^{2}}{3 \xi_{a}(1-\bar{\Gamma})^{2}+4}\right) z^{2} .
$$

Combining (4.26) and (4.27), we obtain

$$
C_{1}=\mathrm{i}\left[-\frac{2}{3} \cot \alpha+\frac{8}{15} \operatorname{Re}-2 W e \bar{E}\left(\frac{3 \xi_{a}(1-\bar{\Gamma})^{2}}{3 \xi_{a}(1-\bar{\Gamma})^{2}+4}\right)\right] .
$$

Therefore, the growth rate of the disturbance is, to this order, equal to

$$
k C_{i}=\frac{8}{15} k^{2}\left[R e-\frac{5}{4} \cot \alpha-\frac{15}{4} W e \bar{E}\left(\frac{3 \xi_{a}(1-\bar{\Gamma})^{2}}{3 \xi_{a}(1-\bar{\Gamma})^{2}+4}\right)\right]
$$

and the critical condition for instability, determined by setting $C_{i}=0$, is found to be

$$
\operatorname{Re}_{c, 0}=\frac{5}{4} \cot \alpha+\frac{15}{4} W e \bar{E}\left(\frac{3 \xi_{a}(1-\bar{\Gamma})^{2}}{3 \xi_{a}(1-\bar{\Gamma})^{2}+4}\right)
$$

a result originally derived by Karapetsas \& Bontozoglou (2013). In the limit of an insoluble surfactant $\left(\xi_{a} \gg 1\right)$ (4.30) is in agreement with the expression given by Wei (2005a) and Pereira \& Kalliadasis (2008a).

It is interesting to note that, for typical constitutive models $\sigma=\sigma(\Gamma)$, the last term on the right-hand side of (4.30) is in general a non-monotonic function of $\bar{\Gamma}$. Thus, moderately soluble surfactants exhibit maximum stabilization at intermediate concentrations. Using as an indicative example the Sheludko model (Sheludko 1967) and a vertical wall $(\cot \alpha=0)$, we find that the dependence of critical $R e$ on surface concentration of surfactant varies parametrically with surfactant solubility as shown in figure 5. At small values of $\xi_{a}$, the maximum in $\operatorname{Re}_{c r}$ occurs around $\bar{\Gamma} \approx 0.27$, whereas with increasing $\xi_{a}$ (decreasing solubility), the maximum gradually drifts towards $\bar{\Gamma}=1$. 


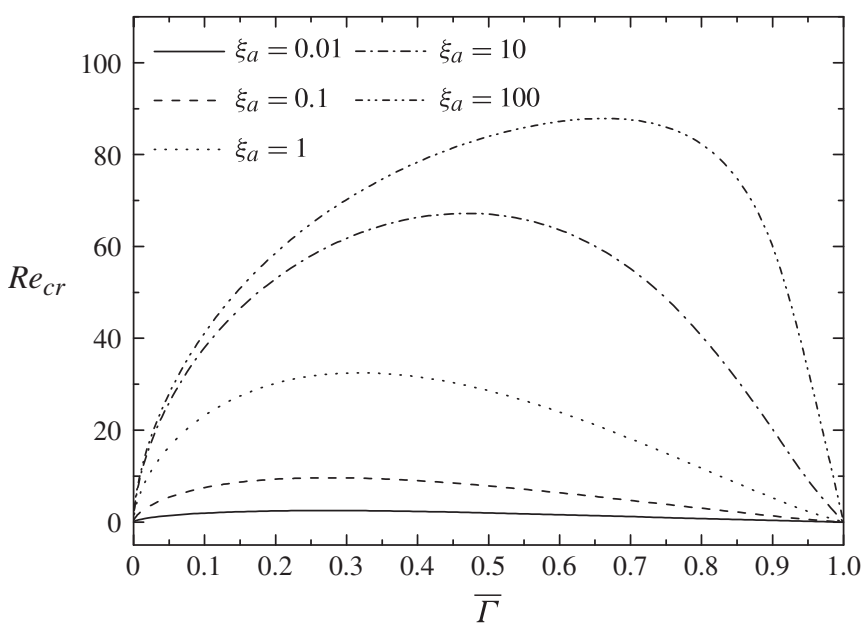

FIGURE 5. Dependence of the critical Reynolds number on the interfacial concentration, $\bar{\Gamma}$ for different values of $\xi_{a}$. The dependence of surface tension on the interfacial concentration was modelled using the equation of state suggested by Sheludko (1967); $\bar{\sigma}=\left[1+\bar{\Gamma}\left(\Sigma^{1 / 3}-1\right)\right]^{-3}$; in this case $\bar{E}$ is given by $\bar{E}=3 \bar{\Gamma}\left(\Sigma^{1 / 3}-1\right)\left[1+\bar{\Gamma}\left(\Sigma^{1 / 3}-1\right)\right]^{-4}$. The rest of the parameters are: $\alpha=90^{\circ}, K a=3000, \Sigma=2 ; \Sigma=\sigma_{c} / \sigma_{m}$, where $\sigma_{c}$ and $\sigma_{m}$ denote the surface tensions of a surfactant-free fluid and that of maximal surfactant concentration, respectively.

\subsection{Higher-order terms}

\subsubsection{Interfacial concentration for non-zero wavenumbers}

The above procedure may be continued to higher orders, at the expense of rapidly increasing algebraic complexity. For example, integrating the $O\left(k^{2}\right)$ terms of $(3.10)$ and combining with the boundary conditions (3.14) at order $O\left(k^{2}\right)$ and (3.15) at order $O\left(k^{1}\right)$, we obtain the first-order amplitude of the interfacial concentration, $\Gamma_{1}$,

$$
\begin{aligned}
\Gamma_{1}= & -\mathrm{i} \frac{\Gamma_{0}^{2}}{\bar{\Gamma}}\left[\frac{8}{9 k_{a} \xi_{a}^{2}(1-\bar{\Gamma})^{3}}+\frac{1}{(1-\bar{\Gamma})^{2} \xi_{a}}\left(\frac{1}{2 P e_{b}}+\frac{37}{105} P e_{b}\right)+\frac{1}{2 P e_{a}}\right. \\
& \left.-\left(\frac{1}{90(1-\bar{\Gamma})^{2} \xi_{a}}-\frac{7}{120}\right) R e+\frac{\bar{E} W e}{3(1-\bar{\Gamma})^{2} \xi_{a}+4}-\frac{\cot \alpha}{12}\right] \\
= & -\mathrm{i} \frac{\Gamma_{0}^{2}}{\bar{\Gamma}} f(\kappa, \mu),
\end{aligned}
$$

where the terms in the squared brackets have been grouped in the function $f(\kappa, \mu)$, with $\kappa=3 k_{a} \xi_{a}(1-\bar{\Gamma})$ and $\mu=3 \xi_{a}(1-\bar{\Gamma})^{2}$. The parametric variation of the function $f(\kappa, \mu)$ is presented in figure 6 for typical values of the dimensionless parameters and for the case of a vertical wall. Equation (4.31) indicates that the effect of adsorption modelling at order $O\left(k^{1}\right)$ and beyond is conveniently described by the parameters $\kappa$ and $\mu$, which are related respectively to the speed of adsorption kinetics and to the solubility of the surfactant. (In particular, we recall from (2.12) that $k_{a} \xi_{a}=k_{1} / U$, therefore, $k_{a} \xi_{a} \gg 1$ means that the interface is always at equilibrium with the bulk, and $k_{a} \xi_{a} \ll 1$ means that the exchange between interface and bulk is negligible, i.e. the interfacial concentration is 'frozen'.) With appropriate selection of the ranges of these 


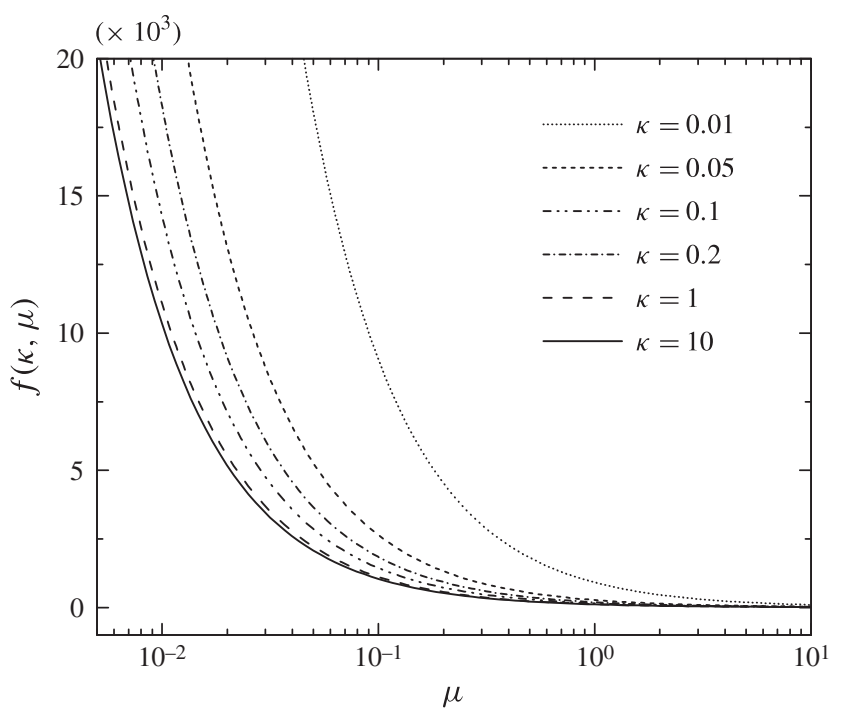

FIGURE 6. Dependence of the function $f(\kappa, \mu)$ on the value of $\mu$ for various values of $\kappa$. Typical values have been used for the rest of the dimensionless parameters: $\operatorname{Re}=100$, $W e=100, P e_{b}=100, P e_{a}=1000, \bar{E}=0.3$ and $\alpha=90^{\circ}$.

parameters, one may examine various particular cases. For example, for an insoluble surfactant $(\kappa \sim O(1), \mu \gg O(1))$, we obtain the expression

$$
\Gamma_{1}=-\mathrm{i} \bar{\Gamma}\left(\frac{7}{30} R e+\frac{2}{P e_{a}}-\frac{1}{3} \cot \alpha\right) .
$$

We note from (4.31) and (4.32) that $\Gamma_{1}$ is purely imaginary and, according to (4.18), so is $c_{1}(z)$ and in particular $c_{1}(1)$. Thus, the phases of the adsorbed and free concentrations at the interface, start at order $O\left(k^{1}\right)$ to deviate from the phase of the interface deformation. More specifically, the phases of the interfacial and bulk concentration, up to this order, are given by the expressions:

$$
\begin{aligned}
\tan \phi_{\Gamma} & =\frac{\operatorname{Im}[\hat{\Gamma}]}{\operatorname{Re}[\hat{\Gamma}]}=\frac{-\mathrm{i} k \Gamma_{1}}{\Gamma_{0}}+O\left(k^{2}\right), \\
\tan \phi_{c} & =\frac{\operatorname{Im}[\hat{c}]}{\operatorname{Re}[\hat{c}]}=\frac{-\mathrm{i} k c_{1}(1)}{c_{0}}+O\left(k^{2}\right) .
\end{aligned}
$$

The direction in which the maximum in $\Gamma^{\prime}$ moves depends on the sign of $\Gamma_{1}$. For an insoluble surfactant, equation (4.32) shows that $\Gamma_{1}$ is negative for all cases of practical interest, therefore the maximum moves ahead of the deformation crest. The same holds also for the general result (4.31), which also contains negative terms. Indeed, the parametric variation of $f(\kappa, \mu)$ depicted in figure 6 shows that for all values of $\kappa$, $f(\kappa, \mu)>0$ and the maximum in surface concentration precedes the deformation crest.

Using (4.18), we can readily show that the phase of the concentration in the bulk is related to that of the interfacial concentration by

$$
\tan \phi_{c}=\tan \phi_{\Gamma}+\frac{4 k}{3(1-\bar{\Gamma}) k_{a} \xi_{a}}+O\left(k^{2}\right)=\tan \phi_{\Gamma}+\frac{4 k}{\kappa}+O\left(k^{2}\right) .
$$


Therefore, $c$ is in phase with $\Gamma$ when the adsorption kinetics are fast enough $(\kappa \gg 1)$, but lags behind it for moderate and slow kinetics. An example of the latter case is provided by the time traces of deformation, flux and concentrations depicted in figure 3 .

\subsubsection{Wave velocity and growth rate for non-zero wavenumbers}

Next, we calculate an improved prediction for the phase velocity, $C_{r}$, valid up to order $O\left(k^{2}\right)$. By using the kinematic boundary condition (3.13) at order $O\left(k^{2}\right)$, we obtain an expression for $C_{2}$, which is real and thus contributes only to $C_{r}$. The final result is

$$
C_{r}=2-k^{2}\left[2+\frac{32}{63} \operatorname{Re}\left(\operatorname{Re}-\frac{5}{4} \cot \alpha\right)+W e \bar{E} \frac{\Gamma_{0}}{\bar{\Gamma}}\left(\frac{\Gamma_{0}}{\bar{\Gamma}} f(\kappa, \mu)-\frac{19}{20} \operatorname{Re}\right)\right]+O\left(k^{4}\right) .
$$

In the limit of a clean liquid $(\bar{E}=0)$, equation (4.36) agrees with the result by Benney (1966). In the limiting case of an insoluble surfactant, equation (4.36) may be further simplified, and after some algebra we obtain

$$
C_{r}=2-k^{2}\left[2+\frac{32}{63} \operatorname{Re}\left(\operatorname{Re}-\frac{5}{4} \cot \alpha-\frac{15}{4} W e \bar{E}\right)+W e \bar{E}\left(\frac{5}{21} \operatorname{Re}+\frac{2}{P e_{a}}-\frac{\cot \alpha}{3}\right)\right] .
$$

It is concluded from (4.37) that for vertical films disturbances of finite wavelength travel slower than the long-wave ones. In particular, it is noted that, from the two terms in parentheses inside the brackets, the first one contains $R e-R e_{c, 0}$ and is thus positive beyond the primary threshold, and for $\alpha=90^{\circ}$ the second contains only two positive terms $\left(5 R e / 21\right.$ and $\left.2 / P e_{a}\right)$.

We follow a similar procedure at order $O\left(k^{3}\right)$ and evaluate $C_{3}$, which is purely imaginary and contributes only to the growth rate. Since the growth rate to this order is given by $k C_{i} \approx C_{1} k^{2}+C_{3} k^{4}$, we determine the critical condition for instability for non-zero wavenumbers $(k \neq 0)$ by setting $C_{i}=0$. As the resulting expression is implicit in $R e$, we derive an analytic solution by positing the expansion

$$
R e_{c}=R e_{c, 0}+k^{2} R e_{c, 2} .
$$

The result in the general case of a surfactant of arbitrary solubility is very cumbersome and will not be presented here. Instead, we quote only the limiting case of a vertical wall $\left(\alpha=90^{\circ}\right)$ and an insoluble surfactant:

$$
R e_{c, 0}=\frac{15}{4} W e \bar{E},
$$

and

$$
R e_{c, 2}=\frac{5 \bar{\sigma}}{4} W e+\left(\frac{4825}{1344}-\frac{1}{P e_{a}^{2}}\right) R e_{c, 0}+\frac{5}{6 P e_{a}} R e_{c, 0}^{2}+\frac{3637}{30888} R e_{c, 0}^{3} .
$$

For a clean fluid $(\bar{E}=0)$, this expression reduces to $R e_{c}=(5 / 4) W e k^{2}$ in agreement with what we know from literature (Benney 1966). With the addition of surfactant, $R e_{c, 0}$ increases from zero and, for typical examples, may eventually reach values of order $10^{2}$. Thus, the last three terms on the right-hand side of (4.40) (and, in particular, the third) dominate the first one, and dictate the behaviour at non-zero wavenumbers. As a consequence, we expect short wave disturbances to stabilize drastically in comparison to the clean fluid. 


\section{Conclusions}

We extended the analysis for the physical mechanism proposed by Kelly et al. (1989) and Smith (1990) for the initiation and growth of a long-wave instability in liquid film flow, in order to account for the presence of a surfactant of arbitrary solubility in the bulk. The zero-order, longitudinal flow perturbation (which is known to result from the perturbation shear stress which develops along the deformed interface) was shown to produce a convective flux that disrupts the uniform interfacial concentration of surfactant. As a consequence of mass conservation, this convective flux builds up an interfacial concentration gradient that, at leading order, is in phase with the deformation of the interface. The stabilizing effect of the resulting Marangoni stresses appears in the first-order flow perturbation, as an additional term assisting gravity in draining fluid from the deformation crest.

The effect of surfactant solubility is apparent in the analytic expression derived for the critical conditions, and indicates that the interfacial concentration gradient, which is responsible for the Marangoni stresses, decreases with increasing solubility. This behaviour is explained by considering mass exchange between the interface and the bulk, which at first order is $90^{\circ}$ out of phase with the deformation, and contributes a flux that opposes the effect of the zero-order flow perturbation. More specifically, it drains surfactant from the node preceding the deformation crest and brings surfactant to the node following it.

It is observed that, for a specific surfactant (constant value of the solubility parameter, $\xi_{a}$ ), the extent of flow stabilization is a non-monotonic function of surfactant concentration at the interface, i.e. the critical Reynolds, $R e_{c, 0}$ number attains maxima at intermediate values of $\Gamma$. This is explained in terms of the aforementioned mass exchange with the bulk solution, which intensifies as the amount of surfactant increases.

Higher-order terms are calculated (at the expense of rapidly escalating algebraic complexity) and provide information on the effect of disturbances of finite wavelength. Apart from surfactant solubility, which remains of central importance, the speed of adsorption/desorption at the interface starts to play a role. This is clearly an effect of finite wavelength, given that for disturbances of infinite length there is always enough time for the bulk to reach equilibrium with the temporally varying interfacial concentration. As the disturbance becomes shorter, the bulk concentration may lag in phase with respect to the interface. The magnitude of this lag depends exclusively on the dimensionless parameter $\xi_{a} k_{a}$, which is the ratio of the characteristic times of convection and adsorption.

Finally, the first correction due to finite wavelength is calculated for the phase velocity of the disturbance and for the critical Reynolds number of the primary instability. The expressions are very cumbersome, and are presented and discussed mainly for the case of an insoluble surfactant. Compared with the results at this order for a clean liquid, the phase velocity is found to decrease with wavenumber, and the critical Reynolds is found to increase strongly.

\section{Acknowledgements}

The authors would like to acknowledge the financial support by the General Secretariat of Research and Technology of Greece under the Action 'Supporting Postdoctoral Researchers' (grant number: PE8/906), cofunded by the European Social Fund and National Resources. 


\section{Appendix.}

Blyth \& Pozrikidis (2004) and Pereira \& Kalliadasis (2008a) have shown for an insoluble surfactant that besides the 'interfacial mode' a 'concentration mode' also exists, which is stable and actually not related to the surfactant property (henceforth, the term 'concentration mode' appears more appropriate than the originally coined 'Marangoni mode'). In the case of a soluble surfactant, we find out that this mode remains stable and its phase velocity is given by

$$
C_{0}=\frac{2+3(1-\bar{\Gamma})^{2} \xi_{a}}{3+3(1-\bar{\Gamma})^{2} \xi_{a}} .
$$

For an insoluble surfactant $\left(\xi_{a} \gg 1\right)$ we get $C_{0}=1$ in agreement with Blyth \& Pozrikidis (2004) and Pereira \& Kalliadasis (2008a).

\section{REFERENCES}

Anshus, B. E. \& ACRIVOS, A. 1967 The effect of surface active agents on the stability characteristics of falling liquid films. Chem. Engng Sci. 22, 389-393.

Benjamin, T. B. 1957 Wave formation in laminar flow down an inclined plane. J. Fluid Mech. 2, $554-573$.

BENJAMin, T. B. 1964 Effects of surface contamination on wave formation in falling liquid films. Arch. Mech. Stos. 16, 615-626.

Benney, D. J. 1966 Long waves on liquid films. J. Math. Phys. 45, 150-155.

BLyth, M. G. \& PozRikidis, C. 2004 Effect of surfactant on the stability of film flow down an inclined plane. J. Fluid Mech. 521, 241-250.

Chang, H. 1994 Wave evolution on a falling film. Annu. Rev. Fluid Mech. 26, 103-136.

Conroy, D. T., Matar, O. K., Craster, R. V. \& Papageorgiou, D. T. 2011 Breakup of an electrified viscous thread with charged surfactants. Phys. Fluids 23 (2), 022103.

Craster, R. V. \& Matar, O. K. 2009 Dynamics and stability of thin liquid films. Rev. Mod. Phys. 81, 1131-1198.

Edmonstone, B. D., Craster, R. V. \& Matar, O. K. 2006 Surfactant-induced fingering phenomena beyond the critical micelle concentration. J. Fluid Mech. 564, 105-138.

Emmert, R. E. \& Pigford, R. L. 1954 A study of gas absorption in falling liquid films. Chem. Engng Prog. 50 (2), 87-93.

Georgantaki, A., Vatteville, J., Vlachogiannis, M. \& Bontozoglou, V. 2011 Measurements of liquid film flow as a function of fluid properties and channel width: evidence for surface-tension-induced long-range transverse coherence. Phys. Rev. E 84, 026325.

Hameed, M., Siegel, M., Young, Y. N., Li, J., Booty, M. R. \& Papageorgiou, D. T. 2008 Influence of insoluble surfactant on the deformation and breakup of a bubble or thread in a viscous fluid. J. Fluid Mech. 594, 307-340.

HINCH, E. J. 1984 A note on the mechanism of the instability at the interface between two shearing fluids. J. Fluid Mech. 144, 463-465.

Jensen, O. E. \& Grotberg, J. B. 1993 The spreading of heat or soluble surfactant along a thin liquid film. Phys. Fluids A: Fluid Dyn. 5, 58-68.

Ji, W. \& Setterwall, F. 1994 On the instabilities of vertical falling liquid films in the presence of surface-active solute. J. Fluid Mech. 278, 297-323.

Kalliadasis, S., Ruyer-Quil, C., Sheid, B. \& Velarde, M. 2012 In Falling Liquid Films, Applied Mathematical Sciences, vol. 176, Springer.

Karapetsas, G. \& Bontozoglou, V. 2013 The primary instability of falling films in the presence of soluble surfactants. J. Fluid Mech. 729, 123-150.

Karapetsas, G., Craster, R. V. \& MAtar, O. K. $2011 a$ On surfactant-enhanced spreading and superspreading of liquid drops on solid surfaces. J. Fluid Mech. 670, 5-37. 
Karapetsas, G., Craster, R. V. \& Matar, O. K. $2011 b$ Surfactant-driven dynamics of liquid lenses. Phys. Fluids 23, 122106.

Kelly, R. E., Goussis, D. A., Lin, S. P. \& Hsu, F. K. 1989 The mechanism for surface wave instability in film flow down an inclined plane. Phys. Fluids A 1, 819-828.

LiN, S. P. 1970 Stabilizing effects of surface-active agents on a film flow. AIChE J. 16 (3), 375-379.

Liu, J., Paul, J. D. \& Gollub, J. P. 1993 A note on the mechanism of the instability at the interface between two shearing fluids. J. Fluid Mech. 250, 69-101.

Oron, A., Davis, S. H. \& BAnKoff, S. G. 1997 Long-scale evolution of thin liquid films. Rev. Mod. Phys. 69, 931-980.

Pereira, A. \& Kalliadasis, S. 2008a Dynamics of a falling film with solutal Marangoni effect. Phys. Rev. E 78, 036312.

Pereira, A. \& Kalliadasis, S. $2008 b$ On the transport equation for an interfacial quantity. Eur. Phys. J. Appl. Phys. 44, 211-214.

Pollak, T., HaAs, A. \& AKsel, N. 2011 Side wall effects on the instability of thin gravity-driven films - from long-wave to short-wave instability. Phys. Fluids 23, 094110.

Sheludko, A. 1967 Thin liquid films. Adv. Colloid Interface Sci. 1, 391-464.

Shkadov, V. Y., Velarde, M. G. \& Shkadova, V. P. 2004 Falling films and the Marangoni effect. Phys. Rev. E 69 (5), 056310.

Smith, M. K. 1990 The mechanism for the long-wave instability in thin liquid films. J. Fluid Mech. 217, 469-485.

Stirba, C. \& Hurt, D. M. 1955 Turbulence in falling liquid films. AIChE J. 1, 178-184.

TAILBY, S. R. \& Portalski, S. 1961 The optimum concentration of surface active agents for the suppression of ripples. Trans. Inst. Chem. 39, 328-336.

WEI, H.-H. $2005 a$ Effect of surfactant on the long-wave instability of a shear-imposed liquid flow down an inclined plane. Phys. Fluids 17, 012103.

WEI, H.-H. 2005b On the flow-induced Marangoni instability due to the presence of surfactant. J. Fluid Mech. 544, 200.

WHITAKER, S. 1964 Effect of surface active agents on the stability of falling liquid films. Ind. Engng Chem. Fundam. 3 (2), 132-142.

Yiantsios, S. G. \& Higgins, B. G. 2010 A mechanism of Marangoni instability in evaporating thin liquid films due to soluble surfactant. Phys. Fluids 22, 022102.

YIH, C.-S. 1963 Stability of liquid flow down an inclined plane. Phys. Fluids 6, 321-334. 